\title{
Hans Blumenberg, L'imitation de la nature et autres essais esthétiques Tenis Trierweiler, dir., Hans Blumenberg. Anthropologie philosophique
} Paris, Hermann, coll. Le Bel Aujourd'hui, 2010 | Paris, Presses universitaires de France, coll. Débats philosophiques, 2010

\section{Tanguy Wuillème}

\section{(2) OpenEdition}

\section{Journals}

Édition électronique

URL : http://journals.openedition.org/questionsdecommunication/2783

DOI : 10.4000/questionsdecommunication. 2783

ISSN : 2259-8901

\section{Éditeur}

Presses universitaires de Lorraine

Édition imprimée

Date de publication : 30 juin 2011

Pagination : $314-317$

ISBN : 978-2-8143-0084-2

ISSN : 1633-5961

\section{Référence électronique}

Tanguy Wuillème, «Hans Blumenberg, L'imitation de la nature et autres essais esthétiques | Denis

Trierweiler, dir., Hans Blumenberg. Anthropologie philosophique », Questions de communication [En ligne], 19 | 2011, mis en ligne le, consulté le 22 septembre 2020. URL : http://journals.openedition.org/ questionsdecommunication/2783; DOI : https://doi.org/10.4000/questionsdecommunication.2783

Ce document a été généré automatiquement le 22 septembre 2020

Tous droits réservés 


\section{Hans Blumenberg, L'imitation de la nature et autres essais esthétiques Denis Trierweiler, dir., Hans Blumenberg. Anthropologie philosophique}

Paris, Hermann, coll. Le Bel Aujourd'hui, 2010 | Paris, Presses universitaires de France, coll. Débats philosophiques, 2010

\section{Tanguy Wuillème}

\section{RÉFÉRENCE}

Hans Blumenberg, L'imitation de la nature et autres essais esthétiques, trad. de l'allemand par Isabelle Kalinowski et Marc de Launay, Paris, Hermann, coll. Le Bel Aujourd'hui, 2010, $170 \mathrm{p}$.

Denis Trierweiler, dir., Hans Blumenberg. Anthropologie philosophique, Paris, Presses universitaires de France, coll. Débats philosophiques, 2010, 162 p.

Les textes qui nous rendent intelligents deviennent de plus en plus rares. L'ultra spécialisation, l'adaptation de styles simplifiés aux attentes des médias ou du public ont fait disparaître l'essai humaniste et percutant. Avec l'attention que porte toute une génération d'analystes à l'œuvre de Hans Blumenberg (1920-1996), on se retrouve en meilleure compagnie. Sous la direction de Denis Trierweiler, sont regroupés un ensemble d'articles consacrés à l'anthropologie philosophique du penseur allemand. Ce recueil complète les approches faites dans une précédente publication («Archives de philosophie ", 2004, cahiers 1 et 2, tome 67) et sur un entreprenant travail de traduction (chez Vrin, au Cerf) d'une œuvre diffuse, massive, encore largement méconnue et non lue. En témoigne cet autre recueil d'articles, cette fois de l'auteur, qui 
donne accès à des textes sur l'esthétique, la rhétorique et la poésie et qui fut publié sous le titre initial de Wirklichkeiten in denen wir leben (que l'on pourrait traduire par Des réalités dans lesquelles nous vivons). Malheureusement, il manque dans la version française un texte important.

2 Si l'ensemble de ces textes et commentaires peuvent paraître disparates, une inquiétude sourd dans l'œuvre d'Hans Blumenberg: une impression de la précarité de toutes choses. Une appréhension générale de la contingence de la vie humaine, du monde, de la situation de l'homme dans le monde, de la raison elle-même et de l'échec possible de toute interprétation de ce qui est et vit. Hans Blumenberg tente de penser dans et contre la phénoménologie, en dialoguant avec Edmund Husserl et Martin Heidegger. Il reproche au premier sa façon de comprendre la réflexion : elle ne saurait être une manière dont la conscience se rapporte à elle-même de façon immédiate, il n'y a pas d'identité entre le penser et l'être-pensé. L'homme n'atteint pas l'objectivité, encore moins une réflexivité directement. Il y a bien une intentionnalité (penser vers), puis une réflexivité (penser ce penser vers) mais elles doivent être complétées par une troisième pensée (qui pense la différence des deux premiers actes de pensée). Hans Blumenberg ne cherche pas seulement à dérouter notre approche de la pensée mais à approfondir son énigme, à se questionner sur la provenance de la réflexion. Pourquoi $\mathrm{y}$-a-t-il quelque chose comme la réflexion ? Quant à Martin Heidegger, il en déplore, là aussi, l'absence de la question de l'homme, de l'anthropologie. Le dasein manque l'homme en chair et en os, celui qui souffre, se questionne, qui veut réussir sa vie. S'il retient du maître de Fribourg l'idée d'angoisse dans la confrontation de l'homme au monde, il ne s'agit pas de retrouver l'essence propre de ce dasein mis au diapason de l'Être. L'angoisse de la précarité demeure, que ne compensent aucune authenticité, aucune réappropriation de soi. L'être humain est cet être sans essence.

De là découle l'enjeu d'une anthropologie philosophique présente dans l'ouvrage d'Hans Blumenberg encore non traduit (et pour cause 918 pages, annoncé au Cerf), Breschreibung des Menschen (Description de l'homme). Il débute avec l'idée que l'homme aurait pu ne pas être et qu'un jour prochain il ne soit plus. Au milieu de cet abîme, se confirment l'indifférence du monde et le souci de s'y confronter. Il ne va pas de soi que l'homme puisse exister. Il est celui qui a donc souci de son auto-conservation et cela le contraint à la réflexion. La réflexion est un «factum» anthropologique lié à l'autoconservation. Il faut entendre ce terme d'auto-conservation non pas avec les yeux du néo-darwinien mais en terme de stabilisation de l'identité. Face aux impressions multiples du monde, comment garantir le soi ? Comprendre est toujours un évitement de la confrontation nue avec les moyens physiques. Ainsi naît la raison, dans l'anthropogenèse. La raison n'est donc pas le propre de l'homme, ce n'est pas lui qui la fait. Elle s'impose à lui.

4 Face à sa vulnérabilité intrinsèque, l'homme peut exister et survivre à condition de ne pas s'engager directement dans la réalité. Il doit s'y référer de manière indirecte, différée. Pour le dire autrement, «métaphoriquement». Ainsi le monde qui, par son caractère infini semblait inaccessible, peut être approché et du même coup amoindrit l'angoisse de notre incapacité à le poser comme une objectivité stable et manipulable. Il s'agit bien d'un affect premier auquel Jean-Claude Monod accorde une attention soutenue dans son article sur «La pulsion de savoir et son destin» (pp.113-140). Il reprend les belles analyses d'Hans Blumenberg sur cette libido sciendi, présentes à la fin de son ouvrage La légitimité des temps modernes (Paris, Gallimard, 1999), texte 
incontournable pour qui veut dorénavant comprendre la querelle de la sécularisation (lire aussi de Jean-Claude Monod, La querelle de la sécularisation de Hegel à Blumenberg, Paris, Vrin, 2002). D'où vient cet appétit si pressant qui nous distingue des animaux ? Quelle est l'histoire de sa reconnaissance comme désir supérieur de contemplation et de quiétude divine (se tenir dans la lumière antique), de sa condamnation chrétiennemédiévale (la curiosité comme vice opposée la venue du Christ qui est toute vérité), de sa réhabilitation moderne (où le monde n'est plus un théâtre avec l'homme comme spectateur mais un tribunal où il en est le juge). Le moderne est celui qui discrimine la mauvaise et la bonne curiosité, celle qui se disperse et rejoint le bizarre de celle qui adopte une méthode, canalise son énergie et s'autocritique. La réflexivité met à distance l'enthousiasme tout en s'aventurant vers le nouveau.

5 Cependant, Hans Blumenberg émet une note pessimiste quant à la contingence de ce désir de savoir : il peut secréter une pulsion de mort car l'individu n'y trouverait jamais l'accomplissement, ni même une maturation et, à la lumière des catastrophes nucléaires ou biologiques à venir, ne serait capable d'aucune limite, d'aucune humilité. Hans Blumenberg montre qu'il ne peut y avoir de consolation possible entre l'homme et ses connaissances qui dorénavant le dépassent individuellement. Pire, la science lui a appris qu'il était voué à une sorte de précarité absolue, sa possible disparition de l'univers avec l'application du second principe de la thermodynamique au cosmos, l'inversion de l'expansion en implosion. Faudrait-il, à la suite de Woody Allen, dire que tout est non sens et tout engagement vanité ? Hans Blumenberg ne répond pas à cette question. Mais il semble que sa réflexion souligne la tentative courageuse de l'homme à affronter sa condition, rendue extrêmement fragile. L'homme est cet être qui pour pouvoir vivre doit constamment s'interpréter lui-même, se demander comment il peut être soi. Il doit donc produire de la pensée, trouver les bons moyens. C'est pourquoi l'histoire humaine est intéressante. Hans Blumenberg, comme le montre bien Pierre Rusch, adopte une approche généalogique, insiste sur la grammaire historique des idées - à chaque époque ses problèmes, son langage, ses notions - mais il montre surtout que les concepts doivent être contextualisés, qu'on y met chaque fois différents contenus. Au fond, ils sont eux-mêmes extrêmement précaires. Le mouvement des idées se détache sur un plan d'évolution plus lente des mots et des images. Il y a des seuils historiques à repérer, des "époques » de transition à approfondir, il existe un principe d'inertie culturelle où les formes survivent à leurs déterminations matérielles. Cette méthode est à l'œuvre dans les textes regroupés sous le titre «l'imitation de la nature ». L'étude éponyme montre comment la modernité a voulu s'affirmer contre cette idée de mimésis de la nature en insistant sur l'idée de créativité humaine, capacité d'invention et d'innovation.

Dans le texte «Paradigme, du point de vue grammatical» (pp.153-158), Hans Blumenberg accepte l'idée de Thomas Kuhn mais il cherche à savoir comment les paradigmes eux-mêmes changent. On peut le savoir car ils sont ancrés dans un cadre constant, la structure de la langue et des flexions grammaticales et il existe bien un même système de besoins élémentaires d'expressions, de conceptions du monde et de soi. La question n'est plus : que pouvons-nous savoir? Mais devient celle-ci : qu'est-ce que nous avons voulu savoir? Quels ont été, dans le passé, les besoins de la pensée ? C'est pourquoi il faut traquer ce que contiennent les concepts mais aussi ce que Hans Blumenberg appellent l'inconceptuabilité : l'indicible-mystique entrevu par Ludwig J. Wittgenstein, l'inconscient de Sigmund Freud, l'usage des métaphores chez Martin Heidegger. Le concept est réifiant, statique, il n'est pas capable de tout ce que la raison 
exige. Le monde est un mouvement et toute approche conceptuelle ne saurait être que provisoire. Hans Blumenberg va se concentrer sur l'étude du mythe, de la métaphore, du symbole, des images comme outils pour l'homme afin de maîtriser la réalité, tous engendrent de la distance, rendent possibles de nouvelles intuitions. Ils sont parties prenantes d'une stratégie fonctionnelle de production de sens. Hans Blumenberg est assez sceptique quant à l'utilisation de ce mot de « sens » qui croit suppléer au thème de l'absurde propre à la pensée européenne après 1945. L'absurde lui-même a du sens, il prospère sur les ruines des promesses de sens non tenues et met à nu la contingence essentielle du monde. Il induit en retour une autre recherche du sens qui cette fois n'est plus naïve mais critique. Elle passe par le détour des métaphores (moyen de fuir l'étrangeté pour aller vers le familier) qui n'est qu'une partie de la rhétorique.

7 Dans un texte central "Approche anthropologique d'une actualité de la rhétorique ", Hans Blumenberg affirme «l'axiome de toute rhétorique, c'est le principe de raison insuffisante. C'est le corrélat de l'anthropologie d'un être à qui l'essentiel manque " (p.115). Face au vide anxiogène du monde, la rhétorique est création d'un espace de manœuvre (pour exemple, la prière est le moyen de persuader Dieu). L'homme séjourne dans la médiation afin de rendre sa vie sensée. Il s'agit bien d'une anthropologie faisant fond sur un individu inquiet, inquiété dès son origine par son existence même, sa place dans le monde, par sa visibilité, qui voit et est visible, qui se voit, qui se voit être vu par les autres et par sa propre conscience et qui n'est jamais sûr de qu'il est ou représente. Cette incertitude a des implications politiques (proie ou prédateur). En découle chez Hans Blumenberg la fragilité des acquis culturels humains et la possibilité de l'échec. "L'homme, écrit Blumenberg dans la La Description de l'homme, est cet être qui, tout comme il peut se rater, est capable de se ressentir comme raté. Le fait qu'il reconnaisse - pas seulement ses semblables - dans le miroir a été décrit comme son unicité ; mais tout autant lui semble-t-il possible de ne pas vouloir l'image de lui qu'il a perçue dans le miroir, parce que, ou bien elle ne correspond à l'image de soi intérieure, ou bien ne satisfait pas l'image idéale que l'on avait voulu réaliser» (Beischreibung, Berlin, Suhrkamp, 2006, p. 681).

8 Face à tous les impératifs de réussite de la vie, d'épanouissement personnel propre à notre modernité ultra-narcissique et auto-satisfaite, face à nos storytelling rétrospectifs et enjolivés, cette interrogation ouvre une béance considérable : l'individu aurait pu être (un) autre, aurait « voulu » être un autre, l'homme est celui qui peut être étranger à lui-même. On retombe sur ce noyau dur de la phénoménologie : ce qu'on croit aller de soi ne va pas de soi et il se pourrait que l'on ait besoin d'une école de l'humilité qui approfondisse nos fragiles certitudes.

\section{AUTEURS}

\section{TANGUY WUILLÈME}

CREM, université Nancy 2 